\title{
Analysis of VISTA expression and function in renal cell carcinoma highlights VISTA as a potential target for immunotherapy
}

\section{Dear Editor,}

Tumors evade immune surveillances, in part via negative regulatory pathways (also called checkpoints) that also regulate immune tolerance to autoimmunity (Thommen et al., 2018). Checkpoint inhibitor therapy, i.e., anti-CTLA-4 and anti-PD-1, has been approved to be an effective therapeutic approach in a variety of cancers (Mariathasan et al., 2018). However, only a subset of cancer patients shows durable responses (Callahan et al., 2016), urging for a broader investigation beyond PD-1 and CTLA-4. Renal cell carcinoma (RCC), the most common kidney cancer, was often considered as an immunogenic tumor based on high levels of T cell infiltration (Finke et al., 1992). However, the infiltrating T cells in RCC were reported to be characterized by a low amount of expanded T cell clonotypes (Sittig et al., 2013). Unexpectedly, the objective response rates to antiPD-1 antibody were $18 \%-31 \%$ in PD-L1 ${ }^{+}$RCC patients vs. 9\%-18\% in PD-L1 ${ }^{-}$patients (Motzer et al., 2015; McDermott et al., 2016). Thus, there is an urgent need for investigation on immune evasion mechanisms in RCC, especially PD-1independent ones.

We thus hypothesized that the low response rate to PD-1 blockade may be caused by co-expression of other checkpoint molecules in the immunosuppressive tumor microenvironment (TME). First, we analyzed the mRNA expression level of several checkpoint molecules in the B7 superfamily through GEPIA using data from TCGA and Oncoprint. We found there was no significant difference in CD274 (encoding PD-L1) expression between RCC tumors and adjacent non-tumoral tissues (Fig. S1A), regardless of RCC types, clear cell RCC (ccRCC), chromophobe RCC (chRCC) or papillary RCC (pRCC). Notably, C10orf54 (encoding VISTA) was significantly upregulated in tumors from patients with CCRCC and downregulated in chRCC tumors compared to adjacent non-tumoral tissues. CD276 (encoding B7-H3) was highly expressed in tumors from patients with $\mathrm{CCRCC}$ as well as pRCC, whereas VTCN1 (encoding B7S1) expression was significantly reduced in all RCC types compared to adjacent non-tumoral tissues. In addition, the expression levels of CD276 and C10orf54 were especially higher than
CD274 in ccRCC tumors (Fig. S1B). These data might underscore the low response rates to PD-1/PD-L1 inhibitors in $\mathrm{CCRCC}$.

To evaluate the expression of the above checkpoint molecules at the protein level in ccRCC accounting for $75 \%$ of RCC, paired tumor and para-tumor tissues $(2 \mathrm{~cm}$ away from tumors) were analyzed by immunofluorescence. The clinical and pathological characteristics of the patients were summarized in Table 1 . Figures $1 \mathrm{~A}$ and $\mathrm{S} 2$ show that VISTA was mostly expressed on $\mathrm{CD} 45^{+}$cells in para-tumors and tumors, consistent with published data that human VISTA is predominantly expressed in hematopoietic tissues and highly expressed within myeloid compartment (Lines et al., 2014; Ni and Dong, 2017b, a). Moreover, the expression level of VISTA in para-tumors was significantly lower than that in tumor sections (Fig. 1B), in line with the expression pattern of VISTA mRNA. In contrast, the expression levels of $\mathrm{B} 7-\mathrm{H} 3$ and $\mathrm{B} 7 \mathrm{~S} 1$ proteins were low in both para-tumors and tumors with no significant difference between the two samples, inconsistent with its mRNA expression pattern (Fig. 1A and 1B). PD-L1 was predominantly expressed by $\mathrm{CD}_{4} 5^{-}$cells (Figs. $1 \mathrm{~A}$ and $\mathrm{S} 2$ ), and there was no significantly difference in PD-L1 expression between para-tumors and tumor tissues (Fig. 1B). To investigate whether ccRCC tumor cells express VISTA, sequential tumor sections were stained by anti-pan-cytokeratin and anti-VISTA, respectively. As shown in Figure 1C, pan-cytokeratin-expressing cells also showed VISTA expression, indicating that ccRCC tumor cells expressed VISTA, but at a relatively lower level.

Since VISTA is mainly expressed on tumor-infiltrated CD $45^{+}$cells, we next sought to identify which subsets of myeloid cells express VISTA. TILs from ccRCC patients were isolated following enzymatic digestion as our lab previously described (Xie et al., 2018). In addition, peripheral blood mononuclear cells (PBMCs) from the same patients were also used as controls. Different surface markers were employed to distinguish myeloid dendritic cells (mDCs, Lin $^{-} \mathrm{HLA}-\mathrm{DR}{ }^{\text {hi }} \mathrm{CD} 11 \mathrm{C}^{+} \mathrm{CD} 123^{-}$), monocytes/macrophages $\left(\mathrm{CD} 14^{+} \mathrm{HLA}^{-D R^{\mathrm{hi}}}\right.$ ) and monocytic myeloid-derived suppressor cells (mMDSCs, CD14 ${ }^{+} H L A-D R^{\text {low/- }}{ }^{-}$. As shown in 
Table 1. Clinical and pathological characteristics of the ccRCC patients

\begin{tabular}{|c|c|c|c|c|}
\hline Patient No. & Gender & Age & TNM stage & $\begin{array}{l}\text { Tumor } \\
\text { stage }\end{array}$ \\
\hline P\#1 & male & 59 & T2NOMO & III \\
\hline P\#2 & male & 65 & T1N0M0 & II \\
\hline P\#3 & male & 48 & T2NOMO & II \\
\hline P\#4 & male & 71 & T2NOMO & II \\
\hline P\#5 & male & 56 & T1N0M0 & II \\
\hline P\#6 & female & 64 & T2N1M0 & III \\
\hline P\#7 & male & 52 & T2N1M0 & III \\
\hline P\#8 & female & 56 & T1N0M0 & II \\
\hline P\#9 & female & 59 & T1N0M0 & II \\
\hline P\#10 & male & 59 & T1N0M0 & II \\
\hline P\#11 & male & 48 & T2NOMO & I \\
\hline P\#12 & male & 45 & T1N0M0 & II \\
\hline P\#15 & male & 58 & T1N0M0 & II \\
\hline P\#16 & female & 70 & T1N0M0 & II \\
\hline P\#17 & male & 49 & T1N0M0 & II \\
\hline P\#18 & male & 82 & T2NOMO & II \\
\hline P\#19 & female & 35 & T1N0M0 & I \\
\hline P\#20 & male & 44 & T1N0M0 & II \\
\hline P\#23 & male & 51 & T1N0M0 & I \\
\hline P\#25 & female & 60 & T2NOMO & II \\
\hline P\#26 & male & 61 & T1N0M0 & I \\
\hline P\#28 & male & 53 & T2N0M1 & III \\
\hline P\#29 & male & 64 & T1N0M0 & II \\
\hline P\#30 & female & 69 & T1N0M0 & II \\
\hline P\#31 & male & 74 & T1N0M0 & I \\
\hline P\#32 & male & 61 & T1N0M0 & I \\
\hline P\#34 & female & 56 & T1N0MO & II \\
\hline P\#35 & female & 51 & T2N0M1 & III \\
\hline P\#37 & female & 76 & T1N0M0 & II \\
\hline P\#41 & female & 58 & T2N0M1 & III \\
\hline P\#43 & female & 77 & T2N1M1 & IV \\
\hline P\#44 & male & 67 & T2N0M1 & II \\
\hline P\#45 & female & 48 & T3NOMO & II \\
\hline P\#46 & male & 52 & T1N0M0 & II \\
\hline P\#47 & female & 68 & T1N0M0 & II \\
\hline P\#48 & male & 29 & T1N0M0 & II \\
\hline P\#50 & female & 54 & T1N0M0 & II \\
\hline P\#51 & male & 46 & T1N0M0 & II \\
\hline P\#52 & female & 53 & T1N0M0 & III \\
\hline P\#53 & male & 64 & T1N0M1 & I \\
\hline P\#54 & female & 73 & T1N0M0 & II \\
\hline P\#55 & male & 70 & T1N0M0 & III \\
\hline
\end{tabular}

Table 1. continued

\begin{tabular}{|c|c|c|c|c|}
\hline Patient No. & Gender & Age & TNM stage & $\begin{array}{l}\text { Tumor } \\
\text { stage }\end{array}$ \\
\hline P\#56 & male & 48 & T1N0M0 & II \\
\hline P\#57 & female & 70 & T1N0M0 & II \\
\hline P\#58 & female & 75 & T1N0M0 & II \\
\hline P\#59 & female & 57 & T1N0M0 & II \\
\hline P\#60 & male & 41 & T3N0M1 & 1 \\
\hline P\#62 & male & 48 & T1N0M0 & II \\
\hline P\#64 & female & 65 & T1N0M0 & I \\
\hline P\#65 & male & 54 & T2NOMO & I \\
\hline P\#66 & male & 50 & T3NOMO & III \\
\hline P\#68 & male & 65 & T3NOMO & II \\
\hline P\#69 & male & 66 & T1N0M0 & I \\
\hline P\#EX-1 & male & 44 & T1N0M0 & II \\
\hline P\#EX-2 & female & 66 & T1N0M0 & I \\
\hline P\#EX-3 & male & 51 & T1N0M0 & $|-| \mid$ \\
\hline P\#EX-4 & female & 51 & T1N0M0 & II \\
\hline P\#EX-5 & male & 85 & $\mathrm{~T} 2 \mathrm{~N} 1 \mathrm{M} 0$ & II \\
\hline P\#EX-6 & male & 35 & T1N0M0 & II \\
\hline P\#EX-7 & male & 50 & T1N0M0 & I \\
\hline P\#EX-8 & female & 53 & T2N0M1 & II \\
\hline P\#EX-9 & male & 61 & T2N0M1 & II \\
\hline P\#EX-10 & male & 49 & T1N0M0 & I \\
\hline P\#EX-11 & male & 54 & T1N0M0 & II \\
\hline P\#EX-12 & female & 60 & T2NOMO & II \\
\hline P\#EX-13 & male & 72 & $\mathrm{~T} 2 \mathrm{n} 0 \mathrm{~m} 0$ & II \\
\hline P\#EX-14 & male & 45 & T1N0M0 & II \\
\hline P\#EX-15 & male & 52 & T1N0MO & I \\
\hline P\#EX-16 & male & 58 & T2N0M1 & III \\
\hline P\#EX-17 & female & 67 & T2NOMO & II \\
\hline
\end{tabular}

Figure 1D-E, the percentages of VISTA-expressing macrophages in the tumors were significantly higher than in PBMCs and para-tumors. However, we did not find significant difference in VISTA expression on mDCs or MDSCs among PBMC, para-tumors and tumors. Consistent with the immunofluorescence results, PD-L1 was not expressed on myeloid cells in ccRCC patients (data not shown). Collectively, the above data not only confirmed the high prevalence of VISTA expression in ccRCC tumors at both mRNA and protein levels, but also revealed the distinct expression patterns of VISTA and PD-L1 in this cancer type.

VISTA is a negative immune checkpoint protein. VISTA expressed on APCs can suppress antigen-specific $T$ cell activation during cognate interactions between APCs and T 

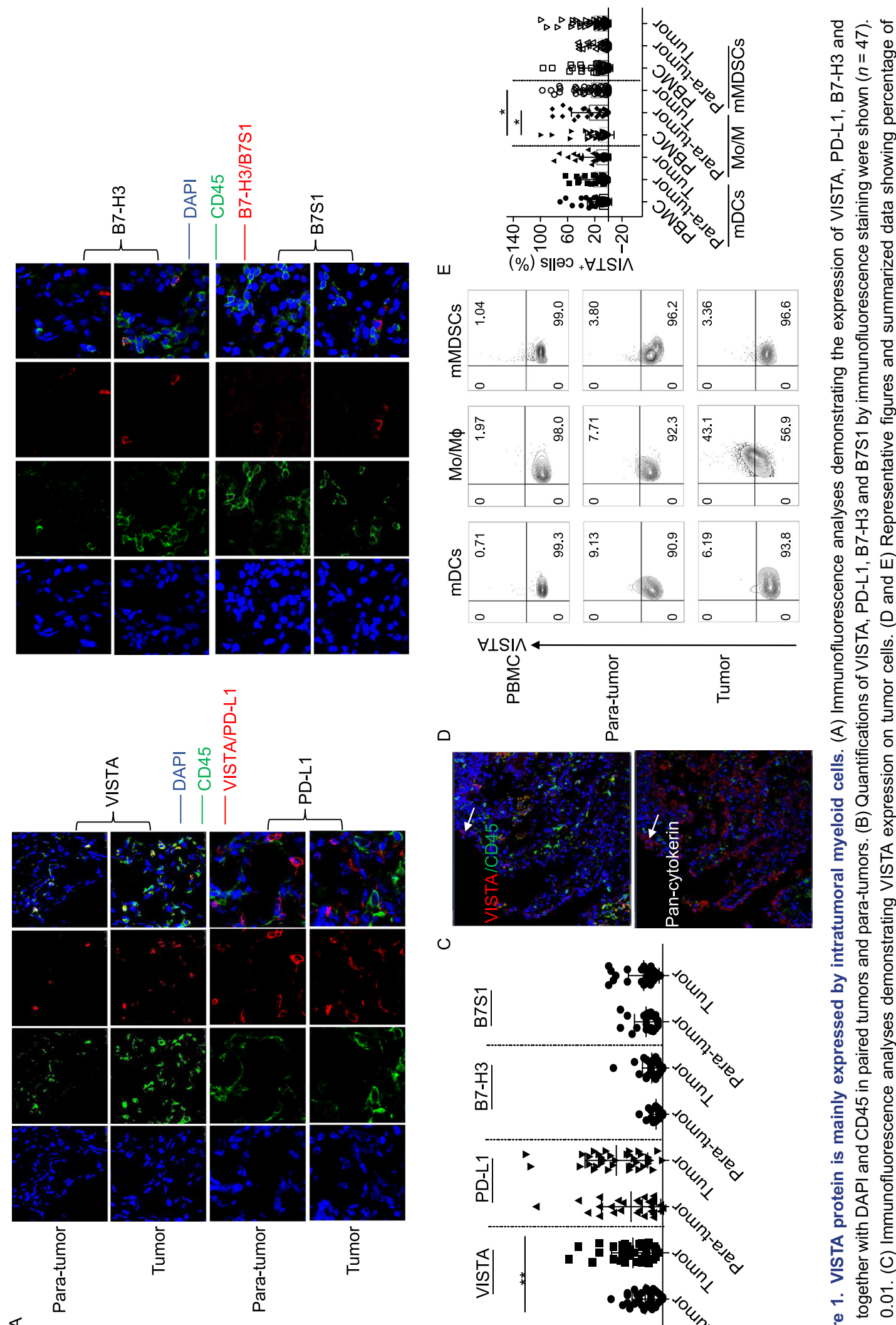

응 ญ 원 这 खं

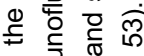
का है

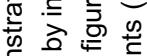
के क $\frac{0}{\bar{\pi}}$ 政 \&

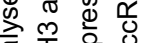
तิ $\frac{m}{1}$ का 동 هัن 过语

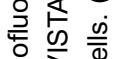
苋员

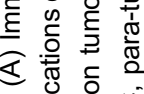
오 늘 들

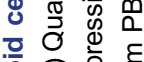
흥 हो त है है u

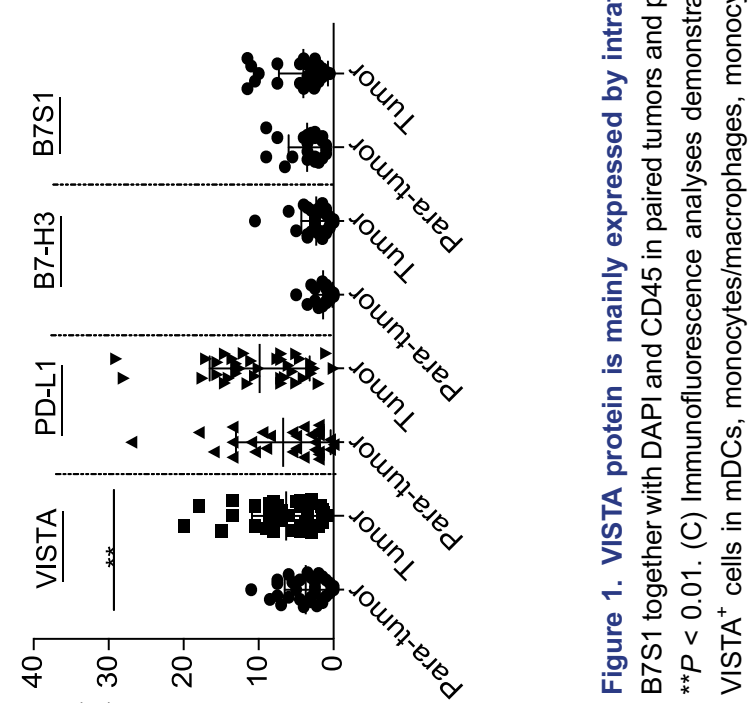



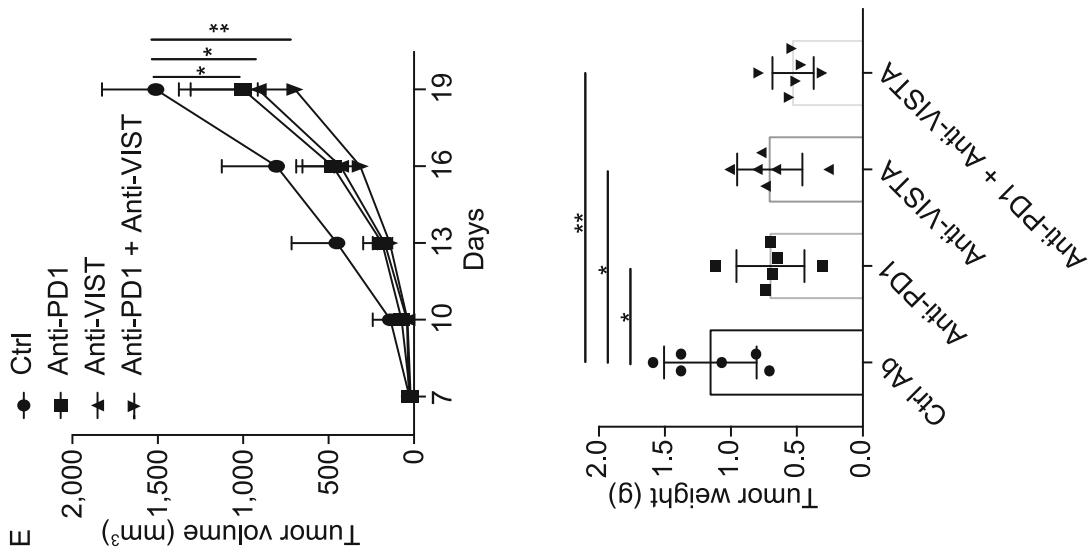

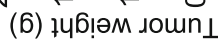

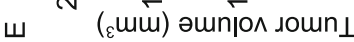

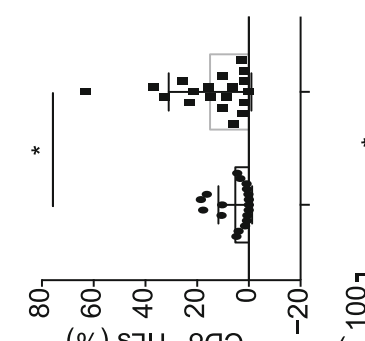

(\%) s기 +800

口+ +u!

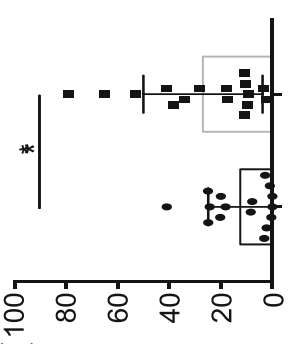

(\%) s기 $\perp+800+e-\mathrm{N} \perp$
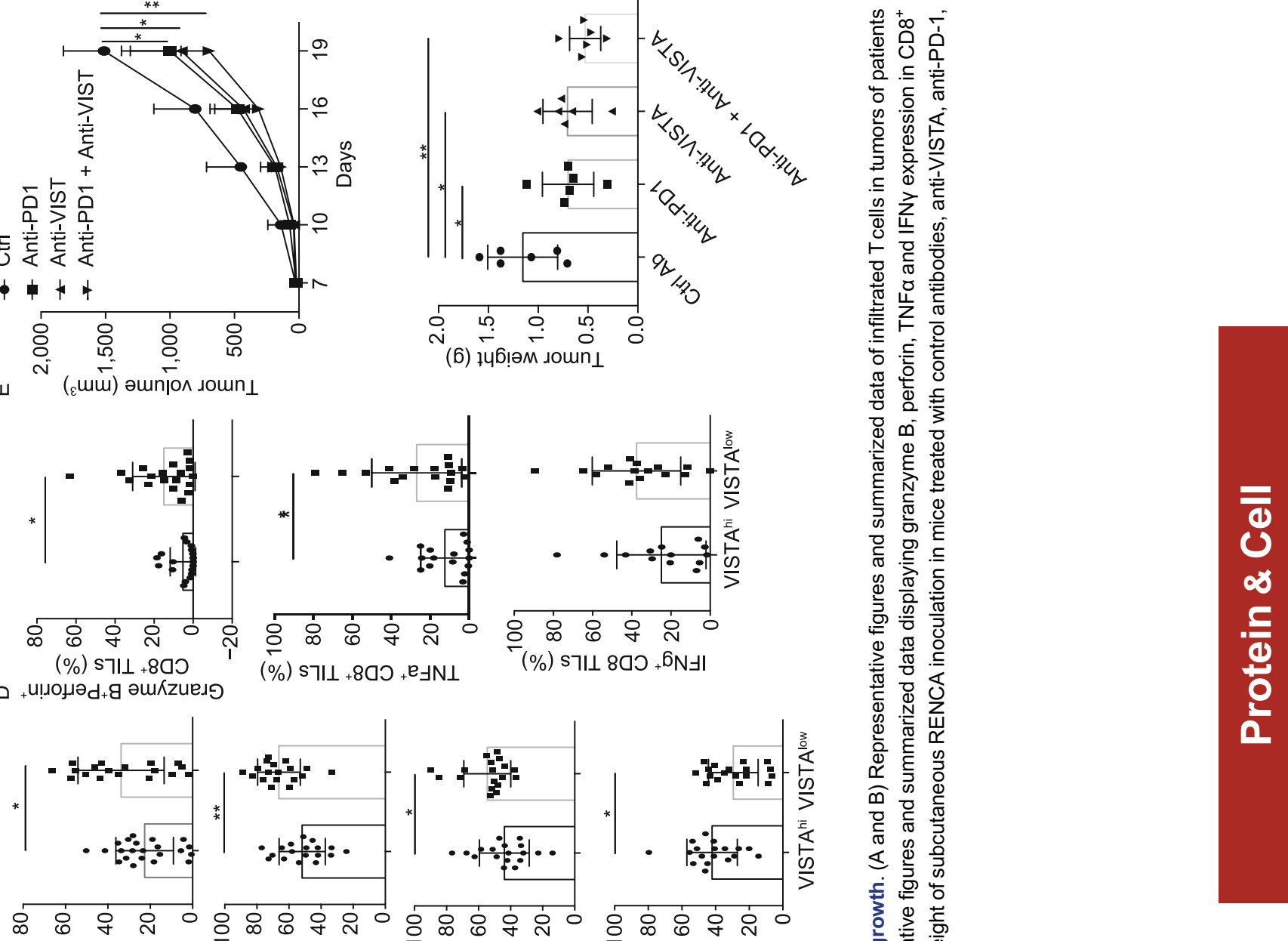

๑ (\%) s기 +staว

(\%) s기 +\&םว
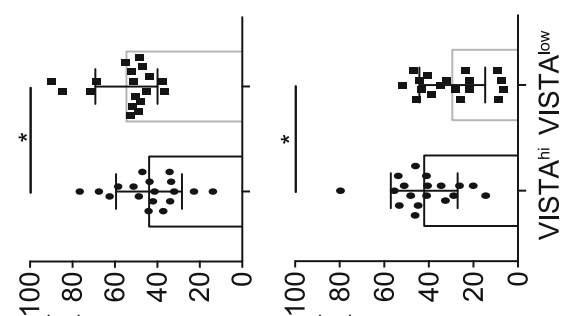

(\%) $s$ 기 +80ว

(\%) sา $\perp+\downarrow 0 \supset$
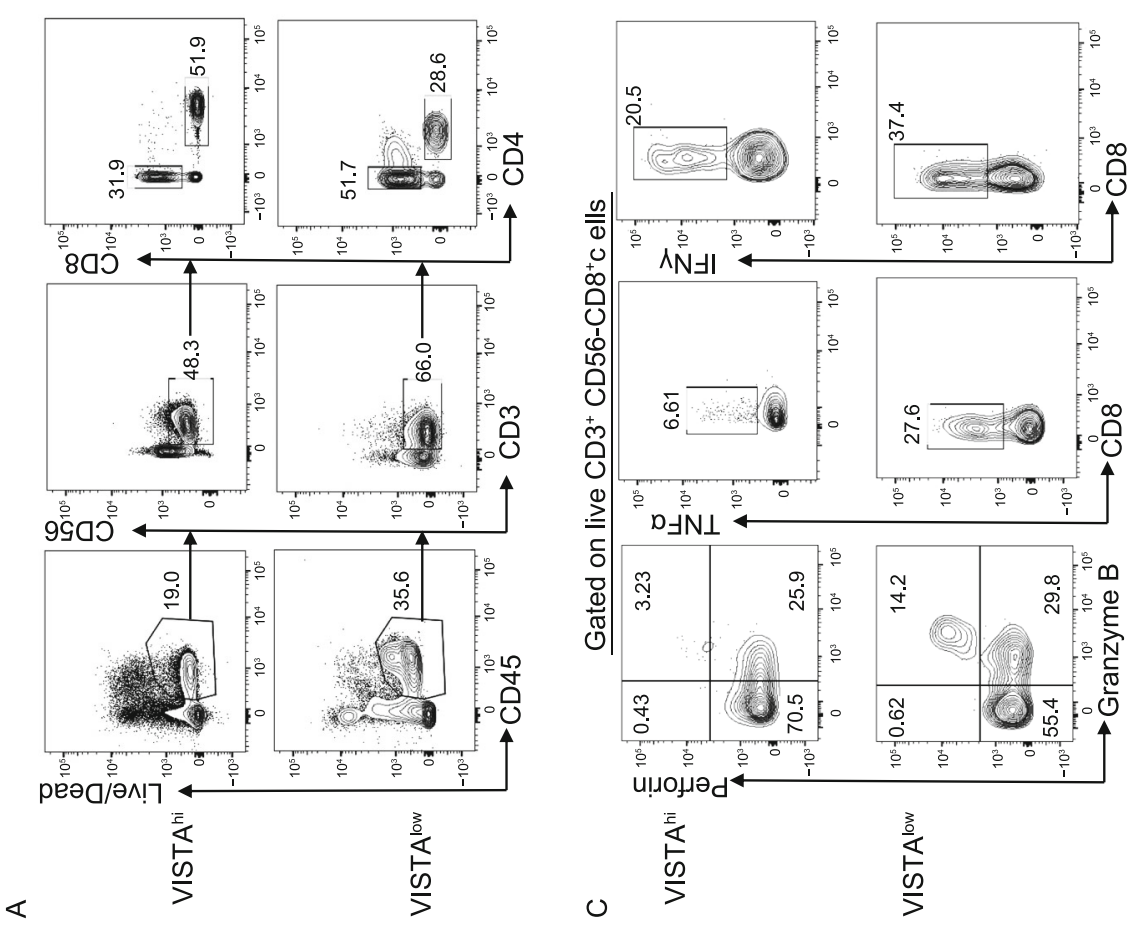

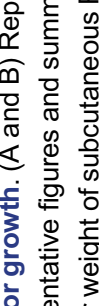

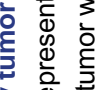

함

음

능 응

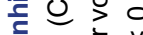
خें है ?

다울

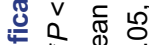

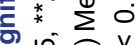

के

í $\dot{0} \dot{0} \dot{0}^{*}$

a

$\varangle$ is $0 \leq$

的此家

范亭 0 .

政 $v$

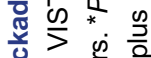

응

ल ख

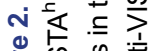

竞零 $\frac{\infty}{\overline{0}}$

은 $\subseteq \stackrel{0}{\square}$ 
cells (Wang et al., 2011). Since CD14 ${ }^{+} \mathrm{HLA}-\mathrm{DR}^{+}$macrophages in the cCRCC tumors expressed higher levels of VISTA, we sought to investigate the relevance of VISTA expression with infiltration and function of $\mathrm{CD} 8^{+}$TILs. To that end, ccRCC patients were divided into two groups VISTA $^{\text {hi }}$ and VISTA ${ }^{\text {low }}$ ) based on the average frequency of VISTA ${ }^{+}$ cells in all CD14 $4^{+}$myeloid cells (The cut-off value is $17.8 \%$ ). Figure $2 \mathrm{~A}$ and $2 \mathrm{~B}$ show that significantly higher frequency of CD45 $5^{+}$TILs as well as $\mathrm{CD} 3^{+} \mathrm{CD} 8^{+}$TILs was found in VIS$\mathrm{TA}^{\text {low }}$ patients than VISTA ${ }^{\text {hi }}$ patients. In contrast, VISTA ${ }^{\text {low }}$ patients had significantly lower fractions of $\mathrm{CD}^{+}$TILs. These findings indicate that VISTA expression on intratumoral $\mathrm{CD} 14^{+}$myeloid cells was negatively associated with the degrees of $\mathrm{CD}^{+} \mathrm{T}$ cell infiltration. Moreover, $\mathrm{CD} 8^{+} \mathrm{TILS}$ in VISTA ${ }^{\text {low }}$ patients displayed higher co-expression of granzyme $B$ and perforin than those in VISTA ${ }^{\text {hi }}$ patients (Fig. 2C and 2D). We also observed a significant increase in $\mathrm{TNFa}^{+} \mathrm{CD}^{+}{ }^{+}$TILs in VISTA ${ }^{\text {low }}$ patients. However, these two groups expressed similar levels of IFNy (Fig. 2C and 2D). These findings indicate that VISTA expression reversely correlates with the cytolytic function of $\mathrm{CD} 8^{+}$TILs, and more closely, with TNFa, but not IFNy, expression. Due to very few NK TILs in some of the ccRCC patients, the correlation between VISTA expression and NK function could not be analyzed.

Having demonstrated that VISTA and PD-L1 may contribute to immune evasion in human ccRCC, we next sought to evaluate the efficacy of anti-VISTA alone or in combination with anti-PD-1 in a syngeneic mouse RCC model, RENCA. Murine VISTA is reported to be primarily expressed by hematopoietic cells and highly upregulated on APCs, but not on B cells, NK cells or granulocytes (Wang et al., 2011; Ni and Dong, 2017b). We found that the RENCA cell line exhibited strong PD-L1 but weak VISTA expression (Fig. S3A). We then investigated the expression patterns of VISTA and PD-L1 in this murine tumor model. Balb/c mice were subcutaneously inoculated with RENCA cells. On day 20 , single cell suspensions of tumors were prepared and stained. VISTA was mainly expressed by CD $45^{+}$TILs, but was detected on very few intratumoral $C D 45^{-}$cells. In line with human counterpart, mouse VISTA was highly expressed on $C D 11 b^{+}, F 4 / 80^{+}$, and $C D 11 c^{+}$myeloid cells in the tumor (Fig. S3B). In contrast to VISTA, PD-L1 was mainly expressed by $\mathrm{CD} 45^{-}$cells and moderate PD-L1 expression was found on $\mathrm{CD} 11 \mathrm{~b}^{+}$and $\mathrm{F} 4 / 80^{+}$myeloid cells (Fig. S3B).

To assess the function of VISTA, Balb/c mice were subcutaneously inoculated with RENCA cells and treated therapeutically with control $\mathrm{Ig}$, anti-VISTA, anti-PD-1, or antiVISTA plus anti-PD-1 antibodies on day 7, 10, 13, 16, and 19 via intraperitoneal injection. Figure $2 \mathrm{E}$ shows that either antiVISTA or anti-PD-1 monotherapy significantly reduced tumor growth compared to control Ig treatment. Although combination of VISTA and PD-1 blockade also resulted in significant tumor reduction, the combined therapy did not elicit a synergistical effect on tumor growth compared with each monotherapy. Notably, we found very few $\mathrm{CD}^{+}$or $\mathrm{CD} 8^{+}$ TILs, but abundant intratumoral myeloid cells (data not shown), consistent with a previous report on RENCA tumor model with disappear of all major $T$ cell subpopulations in late tumors (Yu et al., 2018). Therefore, we could not analyze $\mathrm{CD}^{+}$TILs phenotypically and functionally. In contrast to clinical tumors that contained high percentage of T cells, the RENCA mouse model may not accurately recapitulate the human RCC tumor biology. A xenograft kidney tumor model should be utilized in future study to predict response and investigate the underlying molecular mechanism of this combined therapy.

This study is the first comprehensive phenotypic and functional analysis of VISTA in ccRCC tumors. Higher VISTA is detected in cCRCC tumors than that in non-tumoral tissues at both mRNA and protein levels. However, unlike PD-L1 that is mainly expressed by $\mathrm{CD} 45^{-}$cells, VISTA is highly expressed by intratumoral myeloid cells. ccRCC tumor cells also express VISTA at low levels. Notably, VISTA expression strongly correlated with poor $\mathrm{CD}^{+} \mathrm{T}$ cell responses and blockade of VISTA signaling significantly reduced the growth of murine RENCA RCC model. Taken together, these findings indicate that VISTA functions in suppressing tumor immunity and could serve as a target for immunotherapy in ccRCC.

\section{FOOTNOTES}

This work was supported by grants from Beijing Municipal Science and Technology (Grant No. Z181100001318007 to CD), National key research and development program of China (Grant No. 2016YFC130390 to LN and Grant No. 2018YFC0115905 to JL) and National Natural Science Foundation of China (NSFC) (Grant No. 81570679 to QY).

Shanjuan Hong performed most of the experiments; Yu Feng performed some of the experiments; Qiang Wang, Haizhui Xia, Wei $\mathrm{He}$ and Zhiyin Zhang collected clinical specimens; Qing Yuan and Jian Lu supervised clinical specimens; Ling $\mathrm{Ni}$ and Shanjuan Hong and Chen Dong wrote the manuscript; Jian Lu proofread the manuscript; Chen Dong and Ling Ni designed and supervised the study.

Shanjuan Hong, Qing Yuan, Haizhui Xia, Genzhen Zhu, Yu Feng, Qiang Wang, Zhiyin Zhang, Wei He, Jian Lu, Chen Dong and Ling Ni declare that they have no conflict of interest.

All procedures followed were in accordance with the ethical standards of the responsible committee on human experimentation (institutional and national) and with the Helsinki Declaration of 1975, as revised in 2000 (5). Informed consent was obtained from all patients for being included in the study. 
All institutional and national guidelines for the care and use of laboratory animals were followed.

Shanjuan Hong ${ }^{1}$, Qing Yuan ${ }^{2}$, Haizhui Xia ${ }^{3}$, Genzhen Zhu ${ }^{1}$, Yu Feng ${ }^{1}$, Qiang Wang ${ }^{4}$, Zhiyin Zhang ${ }^{3}$, Wei $\mathrm{He}^{3}$, Jian $\mathrm{Lu}^{3 凶}$, Chen Dong ${ }^{1,5 \bowtie}$, Ling Ni ${ }^{1 凶}$ (D)

${ }^{1}$ Institute for Immunology and School of Medicine, Tsinghua University, Medical Research Building, Beijing 100084, China

2 Division of Urology, The 8th Medical Center of Chinese PLA General Hospital, Beijing 100091, China

${ }^{3}$ Department of Urology, Peking University Third Hospital, Beijing 100191, China

${ }^{4}$ Department of Urology, School of Clinical Medicine, Beijing Tsinghua Changgung Hospital, Tsinghua University, Beijing 102218, China

${ }^{5}$ Beijing Key Lab for Immunological Research on Chronic Diseases, Beijing 100084, China

$\triangle$ Correspondence: lujian@bjmu.edu.cn (J. Lu), chendong@tsinghua.edu.cn (C. Dong), lingni@tsinghua.edu.cn (L. Ni)

\section{OPEN ACCESS}

This article is distributed under the terms of the Creative Commons Attribution 4.0 International License (http://creativecommons.org/ licenses/by/4.0/), which permits unrestricted use, distribution, and reproduction in any medium, provided you give appropriate credit to the original author(s) and the source, provide a link to the Creative Commons license, and indicate if changes were made.

\section{REFERENCES}

Callahan MK, Postow MA, Wolchok JD (2016) Targeting T cell coreceptors for cancer therapy. Immunity 44:1069-1078

Finke JH, Rayman P, Edinger M, Tubbs RR, Stanley J, Klein E, Bukowski R (1992) Characterization of a human renal cell carcinoma specific cytotoxic CD8+ T cell line. J Immunother 1991 (11):1-11

Lines JL, Pantazi E, Mak J, Sempere LF, Wang L, O'Connell S, Ceeraz S, Suriawinata AA, Yan S, Ernstoff MS et al (2014) VISTA is an immune checkpoint molecule for human T cells. Cancer Res 74:1924-1932

Mariathasan S, Turley SJ, Nickles D, Castiglioni A, Yuen K, Wang Y, Kadel EE III, Koeppen H, Astarita JL, Cubas R et al (2018) TGFbeta attenuates tumour response to PD-L1 blockade by contributing to exclusion of T cells. Nature 554:544-548

McDermott DF, Sosman JA, Sznol M, Massard C, Gordon MS, Hamid O, Powderly JD, Infante JR, Fasso M, Wang YV et al (2016) Atezolizumab, an anti-programmed death-ligand 1 antibody, in metastatic renal cell carcinoma: long-term safety, clinical activity, and immune correlates from a phase la study. J Clin Oncol 34:833-842

Motzer RJ, Rini BI, McDermott DF, Redman BG, Kuzel TM, Harrison MR, Vaishampayan UN, Drabkin HA, George S, Logan TF et al (2015) Nivolumab for metastatic renal cell carcinoma: results of a randomized phase II trial. J Clin Oncol 33:1430-1437

Ni L, Dong C (2017a) New B7 family checkpoints in human cancers. Mol Cancer Ther 16:1203-1211

Ni L, Dong C (2017b) New checkpoints in cancer immunotherapy. Immunol Rev 276:52-65

Sittig SP, Kollgaard T, Gronbaek K, Idorn M, Hennenlotter J, Stenzl A, Gouttefangeas C, Thor Straten P (2013) Clonal expansion of renal cell carcinoma-infiltrating $T$ lymphocytes. Oncoimmunology 2:e26014

Thommen DS, Koelzer VH, Herzig P, Roller A, Trefny M, Dimeloe S, Kiialainen A, Hanhart J, Schill C, Hess C et al (2018) A transcriptionally and functionally distinct PD-1(+) CD8(+) T cell pool with predictive potential in non-small-cell lung cancer treated with PD-1 blockade. Nat Med 24:994-1004

Wang L, Rubinstein R, Lines JL, Wasiuk A, Ahonen C, Guo Y, Lu LF, Gondek D, Wang Y, Fava RA et al (2011) VISTA, a novel mouse Ig superfamily ligand that negatively regulates $T$ cell responses. J Exp Med 208:577-592

Xie S, Huang J, Qiao Q, Zang W, Hong S, Tan H, Dong C, Yang Z, Ni $L$ (2018) Expression of the inhibitory B7 family molecule VISTA in human colorectal carcinoma tumors. Cancer Immunol Immunother 67:1685-1694

Yu JW, Bhattacharya S, Yanamandra N, Kilian D, Shi H, Yadavilli S, Katlinskaya Y, Kaczynski H, Conner M, Benson W et al (2018) Tumor-immune profiling of murine syngeneic tumor models as a framework to guide mechanistic studies and predict therapy response in distinct tumor microenvironments. PLoS ONE 13: e0206223

Shanjuan Hong and Qing Yuan contributed equally to this work.

Electronic supplementary material The online version of this article (https://doi.org/10.1007/s13238-019-0642-z) contains supplementary material, which is available to authorized users. 